\title{
Nonlinear eigenvalue problems in fracture mechanics: eigenspectra and eigenfunctions
}

\author{
A.A. Peksheva ${ }^{1}$, L.V. Stepanova ${ }^{1}$ \\ ${ }^{I}$ Samara National Research University, 34 Moskovskoe Shosse, 443086, Samara, Russia
}

\begin{abstract}
The study is aimed at analytical determination of eigenfunctions of the nonlinear eigenvlaue problems following from the crack problems in power law materials under mode III loading and mixed mode (mode I and mode II) loading. The study is based on the perturbation theory technique (the small artificial parameter method) allowing us to find the analytical solution for the eigenfunctions in the closed form in the case of mode III crack problems and to derive the analytical approximations for mixed mode (Mode I and Mode II) crack problems. The method of analytical determination of eigenfunctions of the nonlinear eigenvalue problem is presented.
\end{abstract}

Keywords: crack tip field; antiplane shear; series expansion method; nonlinear eigenvalue problem; eigensolution; eigenspectrum; eigenfunction; closed-form solution

\section{Introduction}

Hutchinson [1,2] and Rice and Rosengren [3] derived the classical Hutchinson-Rice-Rosengren (HRR) stress field in plane stress and plane strain for a crack in power-law hardening materials. They solved the governing nonlinear differential equations for the stress function (describing a nonlinear eigenvalue problem) by a numerical procedure. This solution exclusively describes the dominant singular crack-tip field. Up to now for plane stress and plane strain neither higher order eigensolutions are known nor an analytical solutions for the dominant field is available in the literature [4]. As it is noted in [4] the corresponding antiplane shear problem of a notch with traction free faces in an nonlinear hardening material first was analyzed by Neuber [5] and Rice [6,7] by the use of the hodograph transformation. The brief review of classical results for antiplane deformation of cracked bodies can be found in [4]. Since then researchers have tried to derive the analytical solutions for the antiplane shear problem as well for mode I and mode II crack problems [4 - 25]. Thus in [4] the higher order fields at a notch or a crack tip in the power-law hardening material under mode III loading (longitudinal shear) are studied. The authors derived a closed form solution for the eigenvalues, determining the asymptotic behavior of the fields analytically applying the perturbation technique. It is shown that the eigenvalues of the nonlinear eigenvalue problem solely depend on the eigenvalues of the corresponding linear problem and on the hardening exponent. It is noted that it is valid for all three combinations of homogeneous boundary conditions. A method is derived for constructing the higher order eigensolutions from dominant singular solutions.

The asymptotic stress and strain fields near the crack tip under antiplane shear in an elastic power-law hardening material are developed in [8]. Using an asymptotic expansion and separation of variables for the stress function, a series solution for all of the hardening exponents can be obtained. The stress exponents for the higher order terms are analytically determined; the angular distributions which are governed solely by plastic strains are also analytically obtained. Good agreement with the finite element solutions confirms the proposed approach. It is further demonstrated that the first three terms, controlled by two parameters, can be used to characterize the crack tip stress and strain fields with various hardening exponents. In [8] a series solution with assumed separation of variables form for the stress and strain fields near the crack tip in an elastic power-law hardening material under antiplane shear has been developed. The leading order term is analytically obtained by solving a nonlinear eigenvalue problem. The higher order fields are governed by either linear homogeneous eigenvalue equations or linear nonhomogeneous governing equations. The stress exponents of higher order fields for any hardening exponent are analytically determined. The governing equations for higher order terms which are controlled solely by the plastic strains can also be obtained analytically. However, the governing equations governed by elastic and plastic strains need to be solved numerically. With the analytically determined stress exponents, distinct regions resulting from different strain hardening exponents where the higher order terms up to the fourth order attributed to the plastic strains or elastic and plastic strains can be identified. It has been demonstrated that a truncated three term solution with two parameters accurately characterizes the crack tip stress and strain fields. The paper [9] considers the mechanical fields near the tip of a crack deformed by an anti-plane shear at infinity for a class of nonlinear elastic materials. For brittle materials rupture occurs when a maximal stretch is reached. Taking into account of this critical value, the crack is replaced by a totally damaged zone of finite thickness named a quasicrack. Inside this domain, the stress is identically zero and the shape of the boundary between damaged and undamaged body is found analytically. C. Stolz has determined [9] the shape of the damaged zone under anti-plane shear condition for hyperelastic brittle material. The analytical results are a generalization of preceding results obtained in [10] for brittle materials. The thickness of the damaged zone is determined by the critical strain energy at rupture and the loading. C. Stolz has extended [9] the theory for a more complex constitutive law and recovered results obtained many years ago. The case of power law and its extension on a class of non linear elastic law is discussed with and without brittle damage. With brittle damage one obtains for mode III loading, the geometry of the quasi-crack proposed by Neuber for hardening law has been found. This result is extended to some cases of softening especially for a generalization of the special material introduced in [11]. In [12] the stress and strain fields near the tip of a steady-state growing crack are examined for elastic-viscous materials. A solution to this problem has been originally 
derived by Hui and Riedel [13], with some paradoxes such as the non-dependence of the far fields with respect to the crack growth rate. A two-scale match asymptotic analysis is suggested in [12] to overcome these paradoxes. The scale factor is completely determined by the material properties. The inner scale may be considered as a boundary layer, where the stress field completely described by a serial Fourier analysis. The unit value fits with the Hui and Riedel solution [13].

In [14] it is noted that there exist many nonlinear eigenvalue problems in science and engineering. Nonlinear eigenvalue problems are much more difficult to solve than linear ones. Many nonlinear eigenvalue problems have multiple eigenvalues and eigenfunctions. However, even by means of numerical techniques, it is difficult to find all multiple solutions of a nonlinear differential equation. There are some analytic techniques for nonlinear eigenvalue problems, which are based on either perturbation techniques [15 - 20], or traditional non-perturbation methods such as the Adomian decomposition method [21 - 24], Lyapunov artificial small parameter method [25], and so on. It is well known that perturbation techniques are too strongly dependent upon small physical parameters. Besides, convergence radius of perturbation series is often small, so that perturbation approximations are valid in general only for problems with weak nonlinearity. In [14] a general analytic approach for nonlinear eigenvalue problems is described. Two physical problems are used as examples to show the validity of this approach for eigenvalue problems with either periodic or non-periodic eigenfunctions. Unlike perturbation techniques, this approach is independent of any small physical parameters. Besides, different from all other analytic techniques, it provides a simple way to ensure the convergence of series of eigenvalues and eigenfunctions so that one can always get accurate enough approximations. Finally, unlike all other analytic techniques, this approach provides great freedom to choose an auxiliary linear operator so as to approximate the eigenfunction more effectively by means of better base functions. This approach provides us a new way to investigate eigenvalue problems with strong nonlinearity. In [14] an analytic approach to get series solutions of nonlinear eigenvalue problems is described by means of two examples. This analytic approach is valid for nonlinear eigenvalue problems with either periodic or non-periodic eigenfunctions, and thus is rather general. All of the series solutions agree well with exact or numerical results, and this fact shows the validity of the analytic approach realized in [14]. The author of [14] shows that the analytic approach proposed has some obvious advantages. First of all, unlike perturbation techniques, it is independent of any small physical parameters: it is valid no matter whether or not there exist any small physical parameters in governing equations and/or boundary conditions. Second, different from other traditional techniques, it provides us a simple way to ensure the convergence of series solution of eigenvalue and eigenfunction, so that one can always get accurate enough approximations. Thus, this approach can be applied to solve eigenvalue problems with strong nonlinearity. Third, unlike all other analytic techniques, this approach provides us great freedom to choose an auxiliary linear operator so as to approximate the eigenfunction more effectively by means of better base functions. Therefore, this approach can be widely applied to solve strongly nonlinear eigenvalue problems in science and engineering, no matter whether the corresponding eigenfunction is periodic or not. Analytical approaches to nonlinear eigenvalue problems following from fracture mechanics analysis as well as the perturbation theory methods in general attract many researches in the past and nowadays [9, 12, 26-36].

The present paper is aimed at analytical determination of eigenfunctions of the nonlinear eigenvalue problems arising from the antiplane shear crack problems in power-law materials. The goal of the study is to develop the analytical approach for determination of the eigenfunctions of the nonlinear eigenvalue problems by the perturbation theory methods. The paper continues the perturbation theory method applied for the mode III crack problems in [4, 33-35].

\section{Fundamental equations. Statement of the problem}

Singular fields and higher order fields in the vicinity of the crack in a power-law material under longitudinal shear are investigated in many works [1-4]. The very neat approach for the nonlinear eigenvalue problem has been proposed in [4] where singular fields and higher order fields near a sharp notch in a power-law material under longitudinal shear are analyzed. In [4] using the perturbation theory method the whole set of eigenvalues is determined. A closed form solution for the eigenvalues determining the asymptotic behavior of the fields is analytically derived by applying the perturbation method. However nowadays along with the eigenvalues and along with the eigenspectrum of the problem it is important to know the eigenfunctions corresponding to the eigenvalues derived. In the present paper the closed form solution for the eigenfunctions for the crack tip fields is obtained. It is shown that the asymptotic analysis and methods of summability allow us to derive the analytical solution for the eigenfunctions of the nonlinear eigenvalue problem. The constitutive behavior shall be given by the power law of the Ramberg-Osgood type

$$
\varepsilon_{r z}=3 B \sigma_{e}^{n-1} \sigma_{r z} / 2, \quad \varepsilon_{\theta z}=3 B \sigma_{e}^{n-1} \sigma_{\theta z} / 2, \quad \sigma_{e}=\sqrt{\sigma_{r z}^{2}+\sigma_{\theta z}^{2}}
$$

where $\sigma_{e}$ is the effective stress, and $B$ and the hardening exponent $n$ are materials constants determined experimentally. The equilibrium equation and the compatibility equation in polar coordinates are written as

$$
r \sigma_{r z, r}+\sigma_{\theta z, \theta}+\sigma_{r z}=0, \quad \varepsilon_{r z, \theta}=\left(r \varepsilon_{\theta z}\right)_{, r} .
$$

Introducing the stress function $\chi(r, \theta)$ such as $\sigma_{r z}=r^{-1} \chi,{ }_{\theta}, \sigma_{\theta z}=-\chi,_{r}$ the equilibrium equation is satisfied identically. The asymptotic solution is searched in the separable form

$$
\chi(r, \theta)=r^{s} f(\theta) .
$$

Introducing the asymptotic presentation (3) into (1) and (2) one can obtain the nonlinear ordinary differential equation (NODE)

$$
f_{e}^{2} f^{\prime \prime}+(n-1) f^{\prime 2}\left(f^{\prime \prime}+s^{2} f\right)+[(s-1) n+1] s f_{e}^{2} f=0, \quad f_{e}=\sqrt{\left(f^{\prime}\right)^{2}+(s f)^{2}} \text {. }
$$

The solution of equation (4) should satisfy the conventional traction-free boundary conditions on the crack faces:

$$
f(\theta= \pm \pi)=0 \text {. }
$$

$3^{\text {rd }}$ International conference "Information Technology and Nanotechnology 2017" 
In conjunction with the boundary conditions (5) the nonlinear ordinary differential equation (4) describes a nonlinear eigenvalue problem where the unknown eigenvalue $s$ and the eigenfunction $f(\theta)$ depend on the boundary conditions and the hardening exponent $n$. The unknown eigenvalue $s$ and the eigenfunction $f(\theta)$ should be found as a part of the solution. In [4] the subtle approach allowing us to find the closed form solution for the eigenvalue has been used. Thus, hereafter we will consider that all the eigenvalues are known.

\section{Eigenvalues and eigenfunctions of the antiplane shear problem}

An analytical expression for the eigenfunctions of the nonlinear equation (4) can be derived by applying the perturbation technique. For this purpose, the eigenvalue is represented in the form [4] $s=s_{0}+\varepsilon$, where $s_{0}$ is the eigenvalue of the "undisturbed" linear problem and $\varepsilon$ is the deviation on account of the nonlinearity. Furthermore, the hardening exponent $n$ and the stress function $f(\theta)$ are represented as power series

$$
\begin{aligned}
& n=n_{0}+\varepsilon n_{1}+\varepsilon^{2} n_{2}+\varepsilon^{3} n_{3}+\varepsilon^{4} n_{4}+\varepsilon^{5} n_{5}+\ldots=\sum_{j=0}^{\infty} \varepsilon^{j} n_{j}, \\
& f(\theta)=f_{0}(\theta)+\varepsilon f_{1}(\theta)+\varepsilon^{2} f_{2}(\theta)+\varepsilon^{3} f_{3}(\theta)+\varepsilon^{4} f_{4}(\theta)+\varepsilon^{5} f_{5}(\theta)+\ldots=\sum_{j=0}^{\infty} \varepsilon^{j} f_{j}(\theta)
\end{aligned}
$$

where $n_{0}$ and $f_{0}(\theta)$ are referred to the linear "undisturbed" problem. Introducing the asymptotic expansions (6) and collecting terms of equal power in $\varepsilon$, the following set of linear differential equations is obtained

$$
\begin{aligned}
\varepsilon^{0}: & f_{0}^{\prime \prime}+s_{0}^{2} f_{0}=0 \\
\varepsilon^{1}: & f_{1}^{\prime \prime}+s_{0}^{2} f_{1}=-\left[2+n_{1}\left(s_{0}-1\right)\right] s_{0} f_{0} \\
\varepsilon^{2}: & f_{2}^{\prime \prime}+s_{0}^{2} f_{2}=-\left[n_{1}+n_{2}\left(s_{0}-1\right)\right] s_{0} f_{0}-\left[1+n_{1}\left(s_{0}-1\right)\right] f_{0}-2 s_{0} n_{1} f_{0}^{\prime 2} f_{0} / g_{0} \\
\varepsilon^{3}: & f_{3}^{\prime \prime}+s_{0}^{2} f_{3}=-\left[n_{1}+n_{2}\left(s_{0}-1\right)\right] s_{0} f_{0} g_{1} / g_{0}-\left[1+n_{1}\left(s_{0}-1\right)\right] f_{0} g_{1} / g_{0}-2 s_{0} n_{2} f_{0}^{\prime 2} f_{0} / g_{0}- \\
\quad- & s_{0}\left[n_{2}+n_{3}\left(s_{0}-1\right)\right] f_{0}-\left[n_{1}+n_{2}\left(s_{0}-1\right)\right] f_{0}-\left(f_{2}^{\prime \prime}+s_{0}^{2} f_{2}\right)\left(g_{1}+n_{1} f_{0}^{\prime 2}\right) / g_{0}-n_{1} f_{0}^{\prime 2} f_{0} / g_{0}
\end{aligned}
$$

where $g_{0}=f_{0}^{\prime 2}+s_{0}^{2} f_{0}^{2}, \quad g_{1}=2 f_{0}^{\prime} f_{1}^{\prime}+2 s_{0} f_{0}^{2}+2 s_{0}^{2} f_{0} f_{1}$. The boundary conditions are the conventional traction free conditions on the crack surfaces: $f_{k}(\theta= \pm \pi)=0$. It implies that all the functions $f_{k}(\theta)$ have to satisfy the same condition. It is known that when the boundary value problem for the homogeneous differential equation has a nontrivial solution, the corresponding boundary value problem for inhomogeneous differential equation has a solution if and only if the inhomogeneous part satisfies the solvability condition [20]. The solvability condition permits to find the coefficients $n_{k}$ in equations (6). The coefficients $n_{k}$ have been found and the closed form solution was presented in [4]:

$$
n=1+\frac{s_{0}}{s_{0}-1} \sum_{j=0}^{\infty}\left(\frac{-\left(2 s_{0}-1\right) \varepsilon}{s_{0}\left(s_{0}-1\right)}\right)^{j}-\frac{1}{s_{0}-1} \sum_{j=0}^{\infty}\left(\frac{-\varepsilon}{s_{0}-1}\right)^{j}=\frac{2 s_{0}-1}{\left(2 s_{0}-1\right) s-s_{0}}-\frac{s}{s-1} .
$$

The asymptotic expansion for the hardening exponent when the HRR-type problem $\left(s_{0}=1 / 2\right)$ is considered takes the form

$$
n=1-\frac{1}{s_{0}-1} \sum_{j=0}^{\infty}\left(\frac{-\varepsilon}{s_{0}-1}\right)^{j}=-\frac{s}{s-1} .
$$

It allows us to find the whole spectrum of the eigenvalues

$$
s=\frac{(n+1) \mathrm{s}_{0}+(n-1)\left(2 \mathrm{~s}_{0}-1\right)}{2 n\left(2 \mathrm{~s}_{0}-1\right)}+\frac{\sqrt{\left((n+1) \mathrm{s}_{0}+(n-1)\left(2 \mathrm{~s}_{0}-1\right)\right)^{2}-4 n^{2} s_{0}^{2}\left(2 \mathrm{~s}_{0}-1\right)}}{2 n\left(2 \mathrm{~s}_{0}-1\right)} .
$$

Our aim is to study the possibility to derive the closed form solution for eigenfunctions. To obtain the closed form solution one can solve analytically the system of linear ordinary equations and two point boundary problems for these equations. Further one can analyze the structure of the solution and reveal the general features and inherent properties of the approximate solutions. For this purpose one can analyze the structure of the solutions of each boundary value problem obtained. In the case of a linear material the eigenfunctions and eigenvalues can be easily determined:

$$
f_{0}(\theta)=2 \cos (\theta / 2)
$$

The solution of equation (8) satisfying the traction free boundary conditions $f_{1}(\theta= \pm \pi)=0$ can be written as

$$
f_{1}(\theta)=-n_{1} \cos (\theta / 2)
$$

The solution of equation (9) satisfying the traction free boundary conditions $f_{2}(\theta= \pm \pi)=0$ can be expressed as

$$
f_{2}(\theta)=-\frac{1}{16} \frac{-n_{1}^{2}+n_{1}^{2} \cos ^{2} \theta+8 n_{2} \cos \theta+8 n_{2}}{\cos (\theta / 2)} .
$$

The solution of the two point boundary value problem for equation (10) with the boundary conditions $f_{3}(\theta= \pm \pi)=0$ with respect to function $f_{3}(\theta)$ has the form 


$$
\begin{aligned}
& f_{3}(\theta)=\frac{1}{32} \frac{n_{1}^{3} \sin ^{2} \theta+n_{1}^{3} \cos \theta+3 n_{1}^{3} \cos ^{2}(\theta)-n_{1}^{3} \cos \theta \sin ^{2} \theta-8 n_{1} n_{2} \cos ^{2} \theta-2 n_{1}^{3} \cos ^{3} \theta-4 n_{1} n_{2} \sin ^{2} \theta+8 n_{1} n_{2}-6 n_{1}^{3}}{\cos (\theta / 2)}- \\
& -\frac{1}{32} n_{1} \frac{-2 n_{1}^{2} \cos ^{2} \theta-n_{1}^{2} \sin ^{2} \theta+2 n_{1}^{2}}{\cos (\theta / 2)}+\left[-n_{3}+n_{1} n_{2}+\left(n_{2}-n_{1}^{2}\right) n_{1}\right] \cos (\theta / 2) .
\end{aligned}
$$

Similarly one can find the solution of the boundary value problem for the subsequent function $f_{4}(\theta)$ :

$f_{4}(\theta)=-\frac{n_{1}}{64} \frac{n_{1}^{3} \sin ^{2} \theta+n_{1}^{3} \cos \theta+3 n_{1}^{3} \cos ^{2}(\theta)-n_{1}^{3} \cos \theta \sin ^{2} \theta-8 n_{1} n_{2} \cos ^{2} \theta-2 n_{1}^{3} \cos ^{3} \theta-4 n_{1} n_{2} \sin ^{2} \theta+8 n_{1} n_{2}-6 n_{1}^{3}}{\cos (\theta / 2)}+$ $+\frac{1}{256} \frac{-24 n_{1}^{2} n_{2} \cos ^{3} \theta-4 n_{1}^{4} \sin ^{2} \theta+24 n_{1}^{2} n_{2} \cos \theta-32 n_{1} n_{3} \sin ^{2} \theta+24 n_{1}^{2} n_{2}-24 n_{1}^{2} n_{2} \sin ^{2} \theta \cos \theta+64 n_{1} n_{3}+32 n_{2}^{2}-72 n_{2} n_{1}^{2}}{\cos (\theta / 2)}+$

$+\frac{1}{96} \frac{7 n_{1}^{4}-n_{1}^{4} \cos ^{4} \theta-5 n_{1}^{4} \cos \theta+5 n_{1}^{4} \cos ^{3} \theta}{\cos (\theta / 2)}-\frac{1}{32} \frac{4 n_{1}^{4} \cos ^{2} \theta+8 n_{2}^{2} \cos ^{2} \theta-3 n_{1}^{4} \cos \theta \sin ^{2} \theta+4 n_{2}^{2} \sin ^{2} \theta+16 n_{1} n_{3} \cos ^{2} \theta}{\cos (\theta / 2)}-$.

$-\frac{1}{64} \frac{n_{1}^{4} \sin ^{2} \theta \cos ^{2} \theta}{\cos (\theta / 2)}-\frac{1}{2^{9}} \frac{\left(2 n_{1}^{2}-2 n_{1}^{2} \cos ^{2} \theta-n_{1}^{2} \sin ^{2} \theta\right)^{2}}{\cos (\theta / 2)}-$

$-\frac{1}{4}\left(-n_{2}+n_{1}^{2}\right) \frac{-2 n_{1}^{2} \cos ^{2} \theta-n_{1}^{2} \sin ^{2} \theta+2 n_{1}^{2}}{\cos (\theta / 2)}+\left[-n_{3}+n_{1} n_{3}+\left(n_{2}-n_{1}^{2}\right) n_{2}+\left(n_{3}-2 n_{2} n_{1}+n_{1}^{3}\right) n_{1}\right] \cos (\theta / 2)$.

The solution of the boundary value problem for the function $f_{5}(\theta)$ can be expressed as

$f_{5}(\theta)=\frac{1}{32} \frac{\left(-n_{3}+n_{1} n_{2}+\left(n_{2}-n_{1}^{2}\right) n_{1}\right)\left(2 n_{1}^{2}-2 n_{1}^{2} \cos ^{2} \theta-n_{1}^{2} \sin ^{2} \theta\right)}{\cos (\theta / 2)}$

$-\frac{n_{1}}{256} \frac{12 n_{1}^{2} \sin ^{2} \theta \cos \theta-64 n_{1} n_{3} \cos ^{2} \theta+56 n_{1}^{2} n_{2} \cos ^{2} \theta+64 n_{2} n_{3}+32 n_{2}^{2}-72 n_{1}^{2} n_{2}+(16 \cdot 7 / 3) n_{1}^{4}-16 n_{2}^{2} \sin ^{2} \theta+24 n_{1}^{2} n_{2} \cos \theta}{\cos (\theta / 2)}-$

$-\frac{n_{1}}{256} \frac{32 n_{1} n_{3} \sin ^{2} \theta+16 n_{1}^{4} \cos ^{2} \theta+32 n_{2}^{2} \cos ^{2} \theta+(80 / 3) n_{1}^{4} \sin ^{4} \theta+5 n_{1}^{4} \cos ^{3} \theta-(16 / 3) n_{1}^{4} \cos ^{4} \theta+4 n_{1}^{4} \sin ^{2} \theta}{\cos (\theta / 2)}$

$-\frac{n_{1}}{256} \frac{4 n_{1}^{4} \sin ^{2} \theta \cos ^{2} \theta+12 n_{1}^{2} n_{2} \cos ^{3} \theta+12 n_{1}^{2} n_{2} \sin ^{2} \theta \cos ^{2} \theta+(80 / 3) n_{1}^{4} \cos \theta}{\cos (\theta / 2)}-$

$-\frac{n_{1}}{2^{11}} \frac{\left(2 n_{1}^{2}-2 n_{1}^{2} \cos ^{2} \theta-n_{1}^{2} \sin ^{2} \theta\right)^{2}}{(\cos (\theta / 2))^{3}}-\frac{1}{2^{10}}\left(2 n_{1}^{2}-2 n_{1}^{2} \cos ^{2} \theta-n_{1}^{2} \sin ^{2} \theta\right) \times$

$\times n_{1} \frac{n_{1}^{3} \sin ^{2} \theta+n_{1}^{3} \cos \theta+3 n_{1}^{3} \cos ^{2} \theta-n_{1}^{3} \sin ^{2} \theta \cos \theta-8 n_{1} n_{2} \cos ^{2} \theta-n_{1}^{3} \cos ^{3} \theta-4 n_{1} n_{2} \sin ^{2} \theta-3 n_{1}^{3}+8 n_{1} n_{2}}{(\cos (\theta / 2))^{3}}+$

$+\frac{n_{1}^{5}}{2^{9}} \frac{\cos ^{2} \theta \sin ^{2} \theta-\cos ^{5} \theta-\sin ^{4} \theta \cos \theta+\sin ^{2} \theta}{\cos ^{2}(\theta / 2)}+\frac{n_{1}^{5}}{2^{9} \cdot 3} \frac{47 \cos \theta+19 \cos ^{4} \theta-43}{\cos ^{2}(\theta / 2)}+$

$+\frac{1}{2^{6}} \frac{-n_{1}^{3} n_{2} \sin ^{2} \theta+n_{1}^{3} n_{2} \sin ^{2} \theta \cos \theta-n_{1}^{3} n_{2} \sin ^{2} \theta \cos ^{2} \theta}{\cos ^{2}(\theta / 2)}-\frac{1}{2^{8}} \frac{n_{1}^{3} n_{2} \sin ^{4} \theta+3 n_{1}^{5} \sin ^{2} \theta \cos \theta}{\cos ^{2}(\theta / 2)}+$

$+\frac{1}{2^{6}} \frac{n_{1} n_{2}^{2} \sin ^{2} \theta+3 n_{1}^{3} n_{3} \sin ^{2} \theta-9 n_{1}^{2} n_{3}-9 n_{1} n_{2}^{2}-3 n_{1}^{2} n_{3} \cos ^{3} \theta-3 n_{1} n_{2}^{2} \sin ^{2} \theta \cos \theta-3 n_{1}^{2} n_{3} \sin ^{2} \theta \cos \theta}{\cos ^{2}(\theta / 2)}+$

$+\frac{1}{2^{6}} \frac{n_{1}^{5} \cos ^{2} \theta+3 n_{1}^{2} n_{3} \cos \theta+3 n_{2}^{2} n_{1} \cos \theta+9 n_{2}^{2} n_{1} \cos ^{2} \theta+9 n_{1}^{2} n_{3} \cos ^{2} \theta-3 n_{2}^{2} n_{1} \cos ^{3} \theta}{\cos ^{2}(\theta / 2)}+$

$+\frac{1}{2^{3}} \frac{n_{1} n_{4}+n_{2} n_{3}-n_{1}^{3} n_{2} \cos ^{2} \theta-n_{1} n_{4} \cos ^{2} \theta}{\cos ^{2}(\theta / 2)}+\frac{1}{2^{4}} \frac{5 n_{1}^{3} n_{2} \cos ^{3} \theta-5 n_{2} n_{1}^{3} \cos \theta-n_{1}^{3} n_{2} \cos ^{4} \theta+7 n_{1}^{3} n_{2}}{\cos ^{2}(\theta / 2)}-$

$-\frac{1}{2^{4}} \frac{n_{2} n_{3} \sin ^{2} \theta+n_{1} n_{4} \sin ^{2} \theta}{\cos ^{2}(\theta / 2)}+\frac{1}{2^{7}} \frac{n_{1}^{5} \sin ^{4} \theta-n_{1}^{5} \sin ^{2} \theta \cos ^{3} \theta}{\cos ^{2}(\theta / 2)}+$

$+\left[\left(n_{3}-2 n_{1} n_{2}+n_{1}^{3}\right) n_{2}+\left(\mathrm{n}_{2}-\mathrm{n}_{1}^{2}\right) n_{3}+n_{1} n_{4}-n_{5}+\left(n_{4}-2 n_{1} n_{3}-n_{2}^{2}+3 n_{2} n_{1}^{2}-n_{1}^{4}\right) n_{1}\right] \cos (\theta / 2)+$

$+\frac{1}{2^{6}}\left(-n_{2}+n_{1}^{2}\right) \frac{\left(n_{1}^{3} \sin ^{2} \theta+n_{1}^{3} \cos \theta+3 n_{1}^{3} \cos ^{2} \theta-n_{1}^{3} \sin ^{2} \theta \cos \theta-8 n_{1} n_{2} \cos ^{2} \theta-n_{1}^{3} \cos ^{3} \theta-n_{1} n_{2} \sin ^{2} \theta-3 n_{1}^{3}+n_{1} n_{2}\right)}{\cos (\theta / 2)}$.

The sequence of the solutions obtained has been analyzed carefully. The following perturbation series expansions based on the generalized multinomial theorem [36] are used

$$
\left(\sum_{i=0}^{\infty} \varepsilon^{i} a_{i}\right)^{m}=\left(a_{0}+\varepsilon a_{1}+\varepsilon^{2} a_{2}+\varepsilon^{3} a_{3}+\ldots+\varepsilon^{n} a_{n}+\ldots\right)^{m}=\lambda_{0}+\varepsilon \lambda_{1}+\varepsilon^{2} \lambda_{2}+\varepsilon^{3} \lambda_{3}+\ldots+\varepsilon^{n} \lambda_{n}+\ldots
$$

where $\lambda_{n}$ is the coefficient of the term $\varepsilon^{n}$. The asymptotic expansion of $\left(a_{0}+\varepsilon a_{1}+\varepsilon^{2} a_{2}+\varepsilon^{3} a_{3}+\ldots+\varepsilon^{n} a_{n}+\ldots\right)^{m}$ can be determined as follows [36]: 


$$
\begin{aligned}
& \left(a_{0}+\varepsilon a_{1}+\varepsilon^{2} a_{2}+\varepsilon^{3} a_{3}+\ldots+\varepsilon^{n} a_{n}+\ldots\right)^{m}= \\
& =\sum_{k_{0}=0}^{\infty} \sum_{k_{1}=0}^{k_{0}} \ldots \sum_{k_{n}=0}^{k_{n-1}} \ldots\left(\begin{array}{l}
m \\
k_{0}
\end{array}\right)\left(\begin{array}{l}
k_{0} \\
k_{1}
\end{array}\right) \ldots\left(\begin{array}{l}
k_{n-1} \\
k_{n}
\end{array}\right) \ldots a_{0}^{m-k_{0}}\left(\varepsilon a_{1}\right)^{k_{0}-k_{1}} \ldots\left(\varepsilon^{n} a_{n}\right)^{k_{n-1}-k_{n}} \ldots= \\
& =\sum_{k_{0}=0}^{\infty} \sum_{k_{1}=0}^{k_{0}} \ldots \sum_{k_{n}=0}^{k_{n-1}} \ldots\left(\begin{array}{l}
m \\
k_{0}
\end{array}\right)\left(\begin{array}{l}
k_{0} \\
k_{1}
\end{array}\right) \ldots\left(\begin{array}{l}
k_{n-1} \\
k_{n}
\end{array}\right) \ldots a_{0}^{m-k_{0}} a_{1}^{k_{0}-k_{1}} \ldots a_{n}^{k_{n-1}-k_{n}} \ldots \varepsilon^{0+\left(k_{0}-k_{1}\right)+2\left(k_{1}-k_{2}\right)+\ldots+n\left(k_{n-1}-k_{n}\right)}= \\
& =\sum_{k_{0}=0}^{\infty} \sum_{k_{1}=0}^{k_{0}} \ldots \sum_{k_{n}=0}^{k_{n-1}} \ldots\left(\begin{array}{l}
m \\
k_{0}
\end{array}\right)\left(\begin{array}{l}
k_{0} \\
k_{1}
\end{array}\right) \ldots\left(\begin{array}{l}
k_{n-1} \\
k_{n}
\end{array}\right) \ldots a_{0}^{m-k_{0}} a_{1}^{k_{0}-k_{1}} \ldots a_{n}^{k_{n-1}-k_{n}} \ldots \varepsilon^{k_{0}+k_{1}+k_{2}+\ldots+\mathrm{k}_{n}+\ldots}= \\
& =\lambda_{0}+\varepsilon \lambda_{1}+\varepsilon^{2} \lambda_{2}+\varepsilon^{3} \lambda_{3}+\ldots+\varepsilon^{n} \lambda_{n}+\ldots
\end{aligned}
$$

The coefficient $\lambda_{n}$ can be expressed as

$$
\lambda_{n}=\sum_{k, n}\left(\begin{array}{l}
m \\
k_{0}
\end{array}\right)\left(\begin{array}{l}
k_{0} \\
k_{1}
\end{array}\right) \ldots\left(\begin{array}{l}
k_{n-2} \\
k_{n-1}
\end{array}\right) a_{0}^{m-k_{0}} a_{1}^{k_{0}-k_{1}} \ldots a_{n}^{k_{n-1}}, \quad \sum_{i=0}^{n-1} k_{i}=n, \quad k_{i} \geq k_{i+1} \geq 0 \quad(i=0,1,2, \ldots, n-2) .
$$

The solutions (12) - (16) have been thoroughly analyzed and then the perturbation expansions (17) - (19) are used. This gave the opportunity to derive the general form of the eigenfunction $f_{k}(\theta)$. Having obtained the solutions of the linear ordinary differential equations one can find the sum of the second series expansion in $(6) k=(n-1) /(n+1)$ :

$$
f(\theta)=((n+1) / n) \sqrt{\left[\sqrt{\left(1-k^{2} \sin ^{2} \theta\right)}+\cos \theta\right]\left[\sqrt{\left(1-k^{2} \sin ^{2} \theta\right)}-k \cos \theta\right]^{k} /\left(2(1-k)^{k}\right)} .
$$

\section{Mode I and mixed mode loadings of the cracked specimens}

The objective of this part of the paper is to study the stress singularities at the vicinity of the mixed mode (Mode I and Mode II) crack under plane stress conditions by the approach described above. The governing equations for the power law constitutive relations are transformed to eigenvalue problems of ordinary differential equations (ODEs) based on the assumption that the stress fields are asymptotic near the mixed-mode crack tip. The asymptotic and numerical methods are further developed in the present work to analyze eigenvalue problems of ODEs. Consider a stationary crack in a power-law material under plane stress conditions. Applied loading is accounted as mixed-mode I/II loading. Polar coordinates are introduced and centered at the crack tip. With reference to the polar coordinates the equilibrium equations can be written as

$$
r \sigma_{r r, r}+\sigma_{r \theta, \theta}+\sigma_{r r}-\sigma_{\theta \theta}=0, \quad r \sigma_{r \theta, r}+\sigma_{\theta \theta, \theta}+2 \sigma_{r \theta}=0 .
$$

The compatibility condition has the following form

$$
2\left(r \varepsilon_{r \theta},{ }_{\theta}\right),_{r}=\varepsilon_{r r, \theta \theta}-r \varepsilon_{r r, r}+r\left(r \varepsilon_{\theta \theta}\right),_{r r}
$$

For a material subjected to a power law hardening the constitutive equations for plane stress conditions can be written as follows

$$
\varepsilon_{r r}=B \sigma_{e}^{n-1}\left(2 \sigma_{r r}-\sigma_{\theta \theta}\right) / 2, \quad \varepsilon_{\theta \theta}=B \sigma_{e}^{n-1}\left(2 \sigma_{\theta \theta}-\sigma_{r r}\right) / 2, \quad \varepsilon_{r \theta}=3 B \sigma_{e}^{n-1} \sigma_{r \theta} / 2
$$

where $\sigma_{e}=\sqrt{\sigma_{r r}^{2}+\sigma_{\theta \theta}^{2}-\sigma_{r r} \sigma_{\theta \theta}+3 \sigma_{r \theta}^{2}}$ is the von Mises equivalent stress; $B, n$ are the material constants. It should be noted that in the case considered the analogy between nonlinear elastic behavior and creep holds. That implies that all relations and solutions obtained for a nonlinear elastic (plastic) material with the constitutive equations (3) can be transferred to creep processes with the constitutive relations of Norton's creep law simply by replacing the strains by strain rates. The solution of Eqs. (1) - (3) should satisfy the traditional traction free boundary conditions on the crack surfaces $\sigma_{r \theta}(r, \theta= \pm \pi)=0$, $\sigma_{\theta \theta}(r, \theta= \pm \pi)=0$. The mixed-mode loading can be characterized in terms of the mixity parameter $M^{p}$ which is defined as

$$
M^{p}=(2 / \pi) \operatorname{arctg}\left|\lim _{r \rightarrow 0} \sigma_{\theta \theta}(r, \theta=0) / \sigma_{r \theta}(r, \theta=0)\right| .
$$

The mixity parameter $M^{p}$ equals 0 for pure mode II; 1 for pure mode I, and $0<M^{p}<1$ for different mixities of modes I and II. Thus, for combine-mode fracture the mixity parameter $M^{p}$ completely specifies the near-crack-tip fields for a given value of the hardening exponent $n$. By postulating the Airy stress function $\chi(r, \theta)$ expressed in the polar coordinate system, the stress components state are expressed as: $\sigma_{\theta \theta}=\chi,_{r r}, \sigma_{r r}=\chi,_{r} / r-\chi,_{\theta \theta} / r^{2}, \sigma_{r \theta}=-\left(\chi,_{\theta} / r\right){ }_{r}$. As for the asymptotic stress field at the crack tip $r \rightarrow 0$, one can postulate the following Airy stress function

$$
\chi(r, \theta)=K r^{\lambda+1} f(\theta)
$$

where $K$ is an indeterminate coefficient, $\lambda$ is indeterminate exponent and $f(\theta)$ is an indeterminate function of the polar angle, respectively. In view of the asymptotic presentation (5) the asymptotic stress field at the crack tip is derived as follows $\sigma_{i j}(r, \theta)=K r^{\lambda-1} \sigma_{i j}(\theta)$ or

$$
\sigma_{r r}(r, \theta)=K r^{\lambda-1}\left[(\lambda+1) f(\theta)+f^{\prime \prime}(\theta)\right], \sigma_{\theta \theta}(r, \theta)=K r^{\lambda-1}(\lambda+1) \lambda f(\theta), \sigma_{r \theta}(r, \theta)=-K r^{\lambda-1} \lambda f^{\prime}(\theta)
$$

where $\lambda-1$ denotes the exponent representing the singularity of the stress field, and will be called the stress singularity exponent hereafter. According to (3) the asymptotic strain field as $r \rightarrow 0$ takes the form $\varepsilon_{i j}(r, \theta)=B K^{n} r^{(\lambda-1) n} \varepsilon_{i j}(\theta)$ or in the expanded form 


$$
\begin{aligned}
& \varepsilon_{r r}(r, \theta)=B K^{n} r^{(\lambda-1) n} f_{e}^{n-1}\left[(\lambda+1)(2-\lambda) f(\theta)+2 f^{\prime \prime}(\theta)\right] / 2, \\
& \varepsilon_{\theta \theta}(r, \theta)=B K^{n} r^{(\lambda-1) n} f_{e}^{n-1}\left[(\lambda+1)(2 \lambda-1) f(\theta)-f^{\prime \prime}(\theta)\right] / 2, \varepsilon_{r \theta}(r, \theta)=-3 B K^{n} r^{(\lambda-1) n} f_{e}^{n-1} \lambda f^{\prime}(\theta) / 2 .
\end{aligned}
$$

The compatibility condition (2) results in the nonlinear forth-order ordinary differential equation for the function $f(\theta)$ :

$$
\begin{aligned}
& f^{I V} f_{e}^{2}\left\{(n-1)\left[(\lambda+1)(2-\lambda) f+2 f^{\prime \prime}\right]^{2} / 2+2 f_{e}^{2}\right\}+ \\
& +6[(\lambda-1) n+1] \lambda\left\{(n-1) f_{e}^{2} h f^{\prime}+f_{e}^{4} f^{\prime \prime}\right\}+(n-1)(n-3) h^{2}\left[(\lambda+1)(2-\lambda) f+2 f^{\prime \prime}\right]+ \\
& +(n-1) f_{e}^{2}\left[(\lambda+1)(\lambda+2) f+2 f^{\prime \prime}\right]\left\{\left[(\lambda+1) f^{\prime}+f^{\prime \prime \prime}\right]^{2}+\left[(\lambda+1) f+f^{\prime \prime}\right](\lambda+1) f^{\prime \prime}+\right. \\
& +(\lambda+1)^{2} \lambda^{2}\left(f^{\prime 2}+f f^{\prime \prime}\right)-(\lambda+1)^{2} \lambda f f^{\prime \prime} / 2-\left[(\lambda+1) f^{\prime}+f^{\prime \prime \prime}\right](\lambda+1) \lambda f^{\prime}- \\
& \left.-\frac{1}{2}\left[(\lambda+1) f+f^{\prime \prime}\right](\lambda+1) \lambda f^{\prime \prime}+3 \lambda^{2}\left(f^{\prime \prime 2}+f f^{\prime \prime \prime}\right)\right\}+2(n-1) f_{e}^{2} h\left[(\lambda+1)(2-\lambda) f^{\prime}+2 f^{\prime \prime \prime}\right]+ \\
& +f_{e}^{4}(\lambda+1)(2-\lambda) f^{\prime \prime}-(\lambda-1) n f_{e}^{4}\left[(\lambda+1)(2-\lambda) f+2 f^{\prime \prime}\right]+ \\
& +[(\lambda-1) n+1](\lambda-1) n f_{e}^{4}\left[(\lambda+1)(2 \lambda-1) f-f^{\prime \prime}\right]=0
\end{aligned}
$$

where the following notations are adopted

$$
\begin{aligned}
& f_{e}=\sqrt{\left[(\lambda+1) f+f^{\prime \prime}\right]^{2}+(\lambda+1)^{2} \lambda^{2} f^{2}-\left[(\lambda+1) f+f^{\prime \prime}\right](\lambda+1) \lambda f+3 \lambda^{2} f^{\prime 2}}, \\
& h=\left[(\lambda+1) f+f^{\prime \prime}\right]\left[(\lambda+1) f^{\prime}+f^{\prime \prime \prime}\right]+(\lambda+1)^{2} \lambda^{2} f f^{\prime}-\left[(\lambda+1) f^{\prime}+f^{\prime \prime \prime}\right](\lambda+1) \lambda f / 2-\left[(\lambda+1) f+f^{\prime \prime}\right](\lambda+1) \lambda f^{\prime} / 2+3 \lambda^{2} f^{\prime \prime \prime} .
\end{aligned}
$$

The boundary conditions imposed on the function $f(\theta)$ follow from the traction free boundary conditions on the crack faces:

$$
f(\theta= \pm \pi)=0, \quad f^{\prime}(\theta= \pm \pi)=0 .
$$

One of the effective method for the solution of nonlinear eigenvalue problems is the perturbation theory technique based on the artificially introduced small parameter [14, 16, 20 - 35]. An analytical expression for the eigenvalues of the nonlinear equation (7) can be derived by applying the perturbation theory method. For this purpose the eigenvalue $\lambda$ is split up into $\varepsilon=\lambda-\lambda_{0}$ where $\lambda_{0}$ refers to the "undisturbed" linear problem and $\varepsilon$ is the deviation on account of the nonlinearity. Furthermore, the hardening exponent $n$ and the stress function $f(\theta)$ are represented as power series (6). The set of the boundary value problems for $f_{k}(\theta)$ is obtained:

$$
\begin{aligned}
& \varepsilon^{0}: f_{0}^{I V}+2\left(\lambda_{0}^{2}+1\right) f_{0}^{\prime \prime}+\left(\lambda_{0}^{2}-1\right)^{2} f_{0}=0 \\
& f_{0}(\theta=0)=1, \quad f_{0}^{\prime}(\theta=0)=\left(\lambda_{0}+1\right) / \operatorname{tg}\left(M^{p} \pi / 2\right), \quad f_{0}(\theta=\pi)=0, \quad f_{0}^{\prime}(\theta=\pi)=0, \\
& f_{0}(\theta=-\pi)=0, \quad f_{0}^{\prime}(\theta=-\pi)=0, \quad f_{0}(\theta=0)=1, \quad f_{0}^{\prime}(\theta=0)=\left(\lambda_{0}+1\right) / \operatorname{tg}\left(M^{p} \pi / 2\right), \\
& \varepsilon^{1}: \quad f_{1}^{I V}+2\left(\lambda_{0}^{2}+1\right) f_{1}^{\prime \prime}+\left(\lambda_{0}^{2}-1\right)^{2} f_{1}=-n_{1}\left[x_{0}\left(f_{0}^{I V} x_{0} / 2+w_{0}\right) /\left(2 g_{0}\right)+h_{0}\left(x_{0}^{\prime} g_{0}-x_{0} h_{0}+3 \lambda_{0}^{2} g_{0} f_{0}^{\prime}\right) / g_{0}^{2}\right]- \\
& -f_{0}^{\prime \prime}\left[\left(\lambda_{0}-1\right)\left(4 \lambda_{0}-1\right) n_{1}+8 \lambda_{0}\right] / 2-f_{0}\left(\lambda_{0}^{2}-1\right)\left[\left(\lambda_{0}-1\right)\left(4 \lambda_{0}+1\right) n_{1}+8 \lambda_{0}\right] / 2 \text {, } \\
& f_{1}(\theta=0)=0, \quad f_{1}^{\prime}(\theta=0)=1 / \operatorname{tg}\left(M^{p} \pi / 2\right), \quad f_{1}(\theta=\pi)=0, \quad f_{1}^{\prime}(\theta=\pi)=0, \\
& f_{1}(\theta=-\pi)=0, \quad f_{1}^{\prime}(\theta=-\pi)=0, \quad f_{1}(\theta=0)=0, \quad f_{1}^{\prime}(\theta=0)=1 / \operatorname{tg}\left(M^{p} \pi / 2\right) \\
& \varepsilon^{2}: f_{2}^{I V}+2\left(\lambda_{0}^{2}+1\right) f_{2}^{\prime \prime}+\left(\lambda_{0}^{2}-1\right)^{2} f_{2}=-2 g_{1}\left[f_{1}^{I V}+2\left(\lambda_{0}^{2}+1\right) f_{1}^{\prime \prime}+\left(\lambda_{0}^{2}-1\right)^{2} f_{1}\right] / g_{0}+ \\
& -6 \lambda_{0}\left[2+n_{1}\left(\lambda_{0}-1\right)\right] f_{1}^{\prime \prime}-\left(1-2 \lambda_{0}\right) f_{1}^{\prime \prime}+\left(\lambda_{0}-1\right)\left(1-2 \lambda_{0}\right) f_{1}+\left[1+n_{1}\left(\lambda_{0}-1\right)\right] x_{1}- \\
& -\lambda_{0}\left(\lambda_{0}-1\right)\left(4 \lambda_{0}+1\right) f_{1}-\left(2 \lambda_{0}-1\right)\left[1+n_{1}\left(\lambda_{0}-1\right)\right] y_{1}-n_{1}\left(1-2 \lambda_{0}\right) f_{0}\left(f_{0}^{I V} x_{0}+w_{0}\right) / g_{0} \\
& -6 \lambda_{0}\left[n_{1}+n_{2}\left(\lambda_{0}-1\right)\right] f_{1}^{\prime \prime}-6\left[1+n_{1}\left(\lambda_{0}-1\right)\right] f_{0}^{\prime \prime}+f_{0}^{\prime \prime}-\left(\lambda_{0}-1\right) f_{0}+ \\
& +\left[1+n_{1}\left(\lambda_{0}-1\right)\right]\left(1-2 \lambda_{0}\right) f_{0}-2 \lambda_{0}\left(\lambda_{0}-1\right) f_{0}+\left(2 \lambda_{0}-1\right)\left[1+n_{1}\left(\lambda_{0}-1\right)\right]\left(4 \lambda_{0}+1\right) f_{0}- \\
& -\left(2 \lambda_{0}-1\right)\left[n_{1}+n_{2}\left(\lambda_{0}-1\right)\right] y_{0}-\left[1+n_{1}\left(\lambda_{0}-1\right)\right]^{2} y_{0}+\left[n_{1}+n_{2}\left(\lambda_{0}-1\right)\right] x_{0}- \\
& -2 g_{1}\left\{6_{0}\left[2+n_{1}\left(\lambda_{0}-1\right)\right] f_{0}^{\prime \prime}+\left(1-2 \lambda_{0}\right) f_{0}^{\prime \prime}-\left(\lambda_{0}-1\right)\left(1-2 \lambda_{0}\right) f_{0}-\left[1+n_{1}\left(\lambda_{0}-1\right)\right] x_{0}\right\} / g_{0}- \\
& +\left[1+n_{1}\left(\lambda_{0}-1\right)\right]\left(1-2 \lambda_{0}\right) f_{0}-2 \lambda_{0}\left(\lambda_{0}-1\right) f_{0}+\left(2 \lambda_{0}-1\right)\left[1+n_{1}\left(\lambda_{0}-1\right)\right]\left(4 \lambda_{0}+1\right) f_{0}- \\
& -2 g_{1}\left\{\lambda_{0}\left(\lambda_{0}-1\right)\left(4 \lambda_{0}+1\right) f_{0}+\left(2 \lambda_{0}-1\right)\left[1+n_{1}\left(\lambda_{0}-1\right)\right] y_{0}\right\} / g_{0}-n_{1} 2 h_{1}\left[g_{0} x_{0}^{\prime}-h_{0} x_{0}+3 \lambda_{0}^{2} g_{0} f_{0}^{\prime}\right] / g_{0}^{2}- \\
& -n_{2}\left\{x_{0} g_{0}\left(f_{0}^{I V} x_{0} / 2+w_{0}\right)+2 h_{0}\left[g_{0} x_{0}^{\prime}-h_{0} x_{0}+3 \lambda_{0}^{2} g_{0} f_{0}^{\prime}\right]\right\} / g_{0}^{2}- \\
& -n_{1}\left[x_{0} g_{0}\left(f_{1}^{I V} x_{0} / 2+w_{1}\right)+x_{0} g_{1}\left(f_{0}^{I V} x_{0} / 2+w_{0}\right)+g_{0} x_{1}\left(f_{0}^{I V} x_{0}+w_{0}\right)\right] / g_{0}^{2}+ \\
& -n_{1}\left\{2 h_{0}\left[g_{0} x_{1}^{\prime}-h_{0} x_{1}+3 \lambda_{0}^{2} g_{0} f_{1}^{\prime}\right]+2 h_{0}\left[g_{1} x_{0}^{\prime}-h_{1} x_{0}+3 \lambda_{0}^{2} g_{1} f_{0}^{\prime}\right]\right\} / g_{0}^{2}- \\
& -n_{1}\left\{6 \lambda_{0}\left[2+n_{1}\left(\lambda_{0}-1\right)\right] g_{0} h_{0} f_{0}^{\prime}-2 h_{0}^{2}\left(1-2 \lambda_{0}\right) f_{0}+n_{1} h_{0}^{2} x_{0}+2 g_{0}\left(1-2 \lambda_{0}\right) h_{0} f_{0}^{\prime}\right\} / g_{0}^{2}
\end{aligned}
$$




$$
\begin{aligned}
& \text { Mathematical Modeling / A.A. Peksheva, L.V. Stepanova } \\
& f_{2}(\theta=0)=0, \quad f_{2}^{\prime}(\theta=0)=1 / \operatorname{tg}\left(M^{p} \pi / 2\right), \quad f_{2}(\theta=\pi)=0, \quad f_{2}^{\prime}(\theta=\pi)=0, \\
& f_{2}(\theta=-\pi)=0, \quad f_{2}^{\prime}(\theta=-\pi)=0, \quad f_{2}(\theta=0)=0, \quad f_{2}^{\prime}(\theta=0)=1 / \operatorname{tg}\left(M^{p} \pi / 2\right)
\end{aligned}
$$

where the following notations are used

$$
\begin{aligned}
& x_{k}=\left(\lambda_{0}+1\right)\left(2-\lambda_{0}\right) f_{k}+2 f_{k}^{\prime \prime}, \quad y_{k}=\left(\lambda_{0}+1\right)\left(2 \lambda_{0}-1\right) f_{k}-f_{k}^{\prime \prime}, u_{k}=\left(\lambda_{0}+1\right) f_{k}+f_{k}^{\prime \prime}, \\
& h_{1}=u_{0}\left(u_{1}^{\prime}+f_{0}^{\prime}\right)+u_{0}^{\prime}\left(u_{1}+f_{0}\right)+v_{0}\left[v_{1}^{\prime}+\left(2 \lambda_{0}+1\right) f_{0}^{\prime}\right]+v_{0}^{\prime}\left[v_{1}+\left(2 \lambda_{0}+1\right) f_{0}\right]+3 \lambda_{0} f_{0}^{\prime}\left(\lambda_{0} f_{1}^{\prime \prime}+f_{0}^{\prime \prime}\right)- \\
& -\frac{1}{2} u_{0}^{\prime}\left[v_{1}+\left(2 \lambda_{0}+1\right) f_{0}\right]-\frac{1}{2} u_{0}\left[v_{1}^{\prime}+\left(2 \lambda_{0}+1\right) f_{0}^{\prime}\right]-\frac{1}{2} v_{0}\left(u_{1}^{\prime}+f_{0}^{\prime}\right)-\frac{1}{2} v_{0}^{\prime}\left(u_{1}+f_{0}\right)+3 \lambda_{0} f_{0}^{\prime \prime}\left(\lambda_{0} f_{1}^{\prime}+f_{0}^{\prime}\right), \\
& w_{1}=2 u_{0}^{\prime}\left(u_{1}^{\prime}+f_{0}^{\prime}\right)+u_{0}\left[\left(\lambda_{0}+1\right) f_{1}^{\prime \prime}+f_{0}^{\prime \prime}\right]+\left(\lambda_{0}+1\right) f_{0}^{\prime \prime}\left(u_{1}+f_{0}\right)+2 v_{0}^{\prime}\left[v_{1}^{\prime}+\left(2 \lambda_{0}+1\right) f_{0}^{\prime}\right]+ \\
& +\left(v_{0}^{\prime}-u_{0} / 2\right)\left[v_{1}^{\prime \prime}+\left(2 \lambda_{0}+1\right) f_{0}^{\prime \prime}\right]+v_{0}^{\prime \prime}\left[v_{1}+\left(2 \lambda_{0}+1\right) f_{0}\right]-\left(\lambda_{0}+1\right) f_{0}^{\prime \prime}\left[v_{1}+\left(2 \lambda_{0}+1\right) f_{0}\right] / 2-v_{0}\left[\left(\lambda_{0}+1\right) f_{1}^{\prime \prime}+f_{0}^{\prime \prime}\right] / 2- \\
& -v_{0}^{\prime}\left(u_{1}^{\prime}+f_{0}^{\prime}\right)-u_{0}^{\prime}\left[v_{1}^{\prime}+\left(2 \lambda_{0}+1\right) f_{0}^{\prime}\right]-\frac{1}{2} v_{0}^{\prime \prime}\left(u_{1}+f_{0}\right)+6 \lambda_{0} f_{0}^{\prime \prime}\left(\lambda_{0} f_{1}^{\prime \prime}+f_{0}^{\prime \prime}\right)+3 \lambda_{0} f_{0}^{\prime \prime \prime}\left(\lambda_{0} f_{1}^{\prime}+f_{0}^{\prime}\right)+3 \lambda_{0} f_{0}^{\prime}\left(\lambda_{0} f_{1}^{\prime \prime \prime}+f_{0}^{\prime \prime \prime}\right) .
\end{aligned}
$$

The solution of the fourth-order linear ordinary differential equation (30) with respect to function $f_{0}(\theta)$ satisfying the traction-free boundary conditions has the form: for the crack opening mode I (for symmetric stress fields, pure mode I) $f_{0}^{I}=\beta \cos \alpha \theta-\alpha \cos \beta \theta, \quad \alpha=\lambda_{0}-1, \quad \beta=\lambda_{0}+1$, for the shear crack mode II (the skew-symmetric stress fields, pure mode II) $f_{0}^{I I}=\sin \alpha \theta-\sin \beta \theta$, where the spectrum of the eigenvalues is determined by the characteristic equation $\sin 2 \pi \lambda_{0}=0$, whence one can easily find $\lambda_{0}=m / 2$, where $m$ is an integer. Thus it is shown that an infinite number of eigenvalues exists. In view of the linearity of Eq. (30) for the mixed-mode crack problem the solution is the superposition of the symmetric and antisymmetric parts of the stress field with respect to the crack plane

$$
f_{0}(\theta)=C_{1}(\beta \cos \alpha \theta-\alpha \cos \beta \theta)+C_{2}(\sin \alpha \theta-\sin \beta \theta)
$$

where $C_{1}$ and $C_{2}$ are unknown coefficients which have to be determined from the boundary conditions of the actual crack problem and represent the modes I and II, respectively. In view of (23) the unknown constants $C_{1}$ and $C_{2}$ are related to the mixity parameter $M^{p}=2 \operatorname{arctg}\left[\left(\lambda_{0}+1\right) C_{1} / C_{2}\right] / \pi$. The zeroth-order problem (30) has the nontrivial solution (33), hence the inhomogeneous problems for the functions $f_{1}(\theta)$ and $f_{2}(\theta)$ (13) will not have solutions unless a solvability condition is satisfied $[16,35]$. Therefore, if $\lambda_{0}$ is not an eigenvalue of the homogeneous problem (i.e. the homogeneous problem has only the trivial solution), the inhomogeneous problem has a unique solution for every continuous right hand side $G_{k}(\theta)$ of the differential equation for $f_{k}(\theta), k>0$. On the other hand, if $\lambda_{0}$ is an eigenvalue of the homogeneous problem (i.e. the homogeneous problem has a nontrivial solution), the inhomogeneous problem does not have a solution unless [16, 27, 35]. Following the procedure described in $[16,27,35]$ one can find that the compatibility condition has the form

$$
\int_{-\pi}^{\pi} G_{k}(\theta) u(\theta) d \theta=0
$$

That is, $G_{k}(\theta)$ is orthogonal to the eigenfunction $u(\theta)$, corresponding to the eigenvalue $\lambda_{0}$. These results constitute the socalled Fredholm's theorem: for a given value $\lambda_{0}$, either the inhomogeneous problem has a unique solution for each continuous right hand side of the equation, or else the homogeneous problem has a nontrivial solution $[16,35]$. To determine the solvability condition (34) we use the concept of adjoint problems [16-35]. The boundary value problem (31) is self-adjoint since the differential equation and the boundary conditions of the adjoint problem coincide with the differential equation and boundary conditions of the homogeneous problem (30). Therefore, $u(\theta)=f_{0}(\theta)$, where the function $f_{0}(\theta)$ is determined by Eq. (33). According to Eq. (34) the solvability condition of the boundary value problem (34) has in the expanded form

$$
\begin{aligned}
& \int_{-\pi}^{\pi}\left\{-n_{1}\left[x_{0}\left(f_{0}^{I V} x_{0} / 2+w_{0}\right) /\left(2 g_{0}\right)+h_{0}\left(x_{0}^{\prime} g_{0}-x_{0} h_{0}+3 \lambda_{0}^{2} g_{0} f_{0}^{\prime}\right) / g_{0}^{2}\right]-\right. \\
& \left.-\frac{1}{2} f_{0}^{\prime \prime}\left[\left(\lambda_{0}-1\right)\left(4 \lambda_{0}-1\right) n_{1}+8 \lambda_{0}\right]-\frac{1}{2} f_{0}\left(\lambda_{0}^{2}-1\right)\left[\left(\lambda_{0}-1\right)\left(4 \lambda_{0}+1\right) n_{1}+8 \lambda_{0}\right]\right\} f_{0}(\theta) d \theta=0 .
\end{aligned}
$$

The compatibility condition of the boundary value problem for the function $f_{1}(\theta)$ allows us to find the coefficient $n_{1}$. Having obtained the function $f_{1}(\theta)$, one can determine the unknown function $f_{2}(\theta)$. Using the analogous reasoning, one can formulate the compatibility condition for the solution of the boundary value problem for $f_{2}(\theta)$ and calculate numerically the values of the following coefficient of the asymptotic expansion $n_{2}$ of the hardening exponent $n$ for different values of the mixity parameter.

\section{Results and Discussion}

The perturbation theory method allowed us to find the closed form solution for mode III crack problem. The problem has been reduced to the nonlinear eigenvalue problem and the analytical presentational of the eigenfunction has been obtained by the small parameter method. The asymptotic analysis based on the artificial small parameter method of the perturbation theory provided a possibility to reveal the new stress singularity in the vicinity of the mixed mode crack tip. In the paper the technique 
for numerical determination of the eigenvalues of the nonlinear eigenvalue problem is proposed. Numerical approach allows us to find the eigenfunctions immediately and the results of calculations are shown in Fig. 1. Using this technique the new eigenvalues resulting in the continuous radial stress components at $\theta=0$ are found. It is shown that the method proposed gives the eigenvalues corresponding to the HRR problem in particular cases of mode I and mode II crack problems. The theoretical significance of the present paper is that from the method described here one can clearly know all the mathematically possible distributions of stress singularities at the crack tip under mixed-mode loading. It should be noted that it is important to develop asymptotic analysis methods and their applications for nonlinear eigenvalue problems in solid mechanics [27-37] and, in particular, in nonlinear fracture mechanics and continuum damage mechanics [37] for enunciating newer and better approaches for imparting knowledge on reliable determination of fatigue and fracture behavior. In nonlinear fracture mechanics the
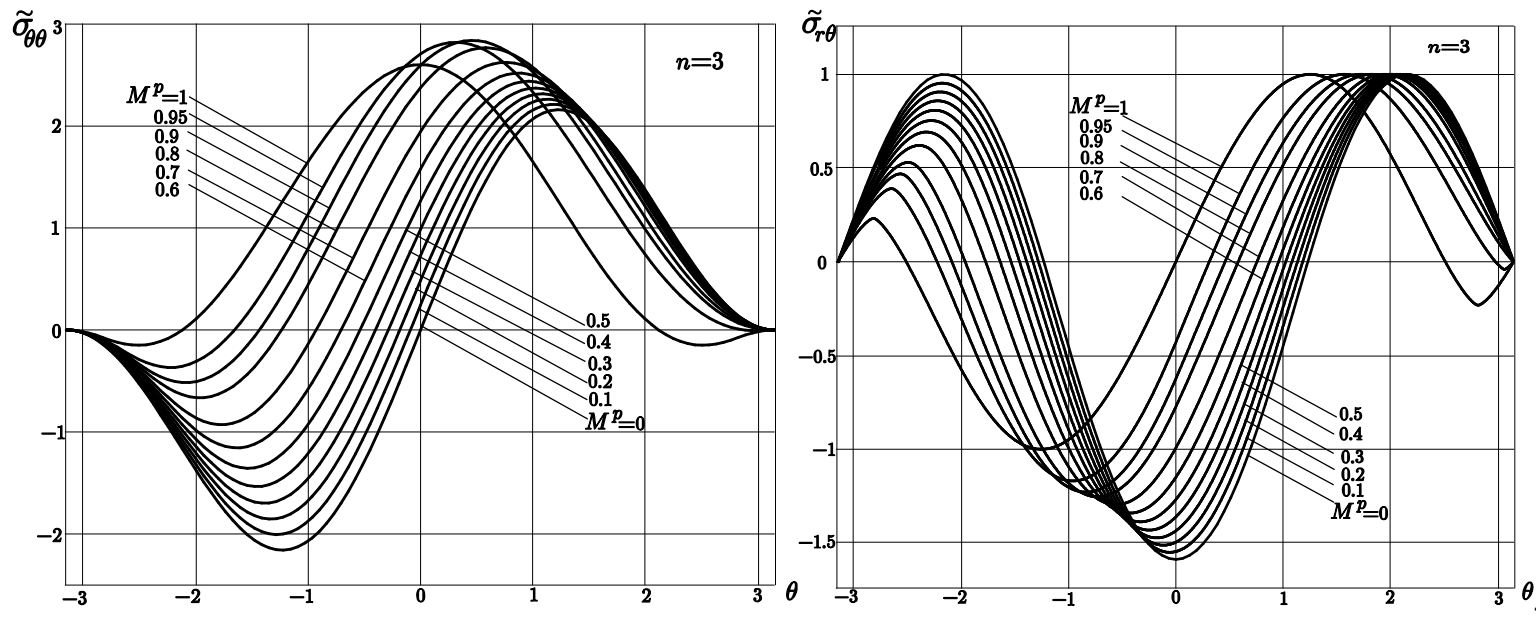

eigenfunction expansion method is one of the most commonly encountered approaches [25-37]. The method leads to nonlinear eigenvalue problems which stipulate the possible distributions of stress singularity at the crack tip and the determination of the whole eigenspectrum requires invoking developed asymptotic and computational techniques and their combinations.

Fig. 1. Eigenfunctions: solution of the nonlinear eigenvalue problem for near crack-tip stress field under mixed-mode loading.

\section{Conclusion}

Using an asymptotic expansion and separation of variables for the stress function a series solution for all hardening exponents is obtained. In the present work the closed form solution for the eigenfunctions for the crack tip fields under antiplane shear is obtained. It is shown that the perturbation method allows us to derive the analytical solution for the eigenfunctions. The approach developed here and the closed-form solution obtained can be used for Mode I, Mode II and mixed mode crack problems for determining the eigenfunctions. It should be noted either that the class of nonlinear eigenvalue problems arising in nonlinear fracture mechanics is essential in connection with creating the multiscale models of fracture with multi-singularities with different orders at the crack point. The singularity representation scheme has to be considered where the local damage at the different scales will be modeled by different orders of the stress singularities. Different stress singularities can be related to different loading type and severity of material damage. In accordance to these models it is necessary to introduce the hierarchy of the zones in the vicinity of the crack tip with dominating role of different stress asymptotic behavior and to realize the matching procedures between different stress asymptotic solutions. The accurate construction of all the intermediate zones with one or other stress asymptotics requires the knowledge of the whole spectrum of eigenvalues and these problems are still open.

\section{References}

[1] Hutchinson JW. Singular behaviour at the end of a tensile crack in a hardening materials. J. Mech. Phys. Solids. 1968; 16: 13-31.

[2] Hutchinson JW. Plastic stress and strain fields at a crack tip. J. Mech. Phys. Solids. 1968; 16: 337-347.

[3] Rice JR, Rosengren GF. Plane strain deformation near a crack tip in a power-law hardening material. J. Mech. Phys. Solids. 1968; 16: 1-2.

[4] Anheuser M, Gross D. Higher order fields at crack and notch tips in power-law materials under longitudinal shear. Archive of Applied Mechanics 1994; 64: $508-518$.

[5] Neuber H. Theory of stress concentration for shear-strained prismatical bodies with arbitrary nonlinear stress-strain law. Trans. ASME/E. Journal of Applied Mechanics 1961; 28: 544-550.

[6] Rice JR. Contained plastic deformation near cracks and notches under longitudinal shear. International Journal of Fracture Mechanics 1966 ; 2 : $426-447$.

[7] Rice JR. Stresses due to a sharp notch in a work-hardening elastic-plastic material loaded by longitudinal shear. Trans. ASME/E. Journal of Applied Mechanics 1967; 34: 287-298.

[8] Yang S, Yuan FG, Cai X. Higher order asymptotic elastic-plastic crack-tip fields under antiplane shear. Engineering Fracture Mechanics 1996; 54(3): 405422.

[9] Stolz C. Asymptotic fields ahead a crack for a class of non linear materials under mode III. Mechanics of Materials 2015; 90: $102-110$.

[10] Bui HD, Ehrlacher A. Propagation dynamique d'une zone endommagee dans un solide elastique fragile en mode III et en regime permanent. C.R. Acad. Sci. Paris, Ser. B 1980; 290: 273-276.

[11] Abeyaratne R. Discontinuous deformation gradients away from the tip of a crack in anti-plane shear. J. Elast. 1981; 11: 373-393.

[12] Abdelmoula R, Debruyne G. Analysis of the stress and strain fields near the crack tip of a steady-state growing crack in an elastic-viscous medium: The Hui-Riedel problem revisited by means of method of matched asymptotic expansions. Comptes Rendus Mechanique 2016; 344: 613-622.

[13] Hui CY, Riedel H. The asymptotic stress and strain field near the tip of a growing crack under creep conditions. Int. J. Fract. 1981; 17: 409-425.

[14] Liao S. Series solution of nonlinear eigenvalue problems by means of the homotopy analysis method. Nonlinear Analysis: Real World Applications 2009; 10: $2455-2470$.

[15] Cole JD. Perturbation Methods in Applied Mathematics. Blaisdell Publishing Company, Waltham, Massachusetts, 1968. 
[16] Nayfeh AH. Introduction to Perturbation Techniques. John Wiley \& Sons, New York, 1981.

[17] Murdock A. Perturbations: Theory and Methods. John Wiley \& Sons, New York, 1991.

[18] Bush AW. Perturbation Methods for Engineers and Scientists. CRC Press Library of Engineering Mathematics, CRC Press, Boca Raton, 1992.

[19] Kevorkian J, Cole JD. Multiple Scales and Singular Perturbation Methods. Applied Mathematical Sciences $1995 ; 114$.

[20] Nayfeh AH. Perturbation Methods. John Wiley \& Sons, New York, 2000.

[21] Adomian G. Nonlinear stochastic differential equations. J. Math. Anal. Appl. 1976; 55: 441-452.

[22] Rach R. On the Adomian method and comparisons with Picard's method. J. Math. Anal. Appl. 1984; 10: 139-159.

[23] Adomian G, Rach R. On the solution of algebraic equations by the decomposition method. Math. Anal. Appl. 1985 ; $105(1)$ : $141-166$.

[24] Adomian G. A review of the decomposition method and some recent results for nonlinear equations. Comput. Math. Appl. 1991; 21: 101-127.

[25] Lyapunov AM. General Problem on Stability of Motion. Taylor \& Francis, London, 1992.

[26] Ehrlacher A, Markenscoff X. Duality, Symmetry and Symmetry Lost in Solid Mechanics. Press des Ponts, Paris, 2011.

[27] Stepanova LV, Igonin SA. Asymptotics of the near-crack-tip stress field of a growing fatigue crack in damaged materials: Numerical experiment and analytical solution. Numerical Analysis and Applications 2015; 8(2): 168-181.

[28] Stepanova LV, Adylina EM. Stress-strain state in the vicinity of a crack tip under mixed loading. Journal of Applied Mechanics and Technical Physics 2014; 55(5): 885-895.

[29] Bui HD. Fracture Mechanics: Inverse problems and Solutions. Dordrecht: Springer, 2006.

[31] Stepanova LV, Yakovleva EM. Mixed-mode loading of the cracked plate under plane stress conditions. PNRPU Mechanics Bulletin $2014 ; 3: 129-162$.

[32] Paulsen W. Asymptotic Analysis and Perturbation Theory. Boca Raton, London, New York: CRC Press, 2014.

[33] Stepanova LV. Eigenvalues of the antiplane-shear crack problem for a power-law material. Journal of Applied Mechanics and Technical Physics 2008; 49(1): 142-147.

[34] Stepanova LV. Eigenspectra and orders of stress singularity at a mode I crack tip for a power-law medium. Comptes Rendus - Mecanique 2008; 336(1-2): $232-237$.

[35] Stepanova L, Yakovleva E. Stress-strain state near the crack tip under mixed-mode loading: Asymptotic approach and numerical solutions of nonlinear eigenvalue problems. AIP Conference Proceedings 2016; 1785: 030030.

[36] Qiu Z, Zheng Y. Predicting fatigue crack growth evolution via perturbation series expansion method based on the generalized multinomial theorem. Theoretical and Applied Fracture Mechanics 2016; 86: 361-369.

[37] Stepanova LV, Fedina MYe. Self-similar solution of a tensile crack problem in a coupled formulation. Journal of Applied Mathematics and Mechanics 2008; 72(3): 360-368. 